\title{
Probing Late Neutrino Mass Properties with Supernova Neutrinos
}

\author{
Joseph Baker, ${ }^{1}$ Haim Goldberg, ${ }^{2}$ Gilad Perez, ${ }^{3,4}$ and Ina Sarcevic ${ }^{1}$ \\ ${ }^{1}$ Department of Physics, University of Arizona, Tucson AZ 85721 \\ ${ }^{2}$ Department of Physics, Northeastern University, Boston, MA 02115 \\ ${ }^{3}$ Theoretical Physics Group, Ernest Orlando Lawrence Berkeley National Laboratory, \\ University of California, Berkeley, CA 94720 \\ ${ }^{4}$ C.N. Yang Institute for Theoretical Physics State \\ University of New York Stony Brook, NY 11794-3840
}

(Dated: April 9, 2018)

\begin{abstract}
Models of late-time neutrino mass generation contain new interactions of the cosmic background neutrinos with supernova relic neutrinos (SRNs). Exchange of an on-shell light scalar may lead to significant modification of the differential SRN flux observed at earth. We consider an Abelian U(1) model for generating neutrino masses at low scales, and show that there are cases for which the changes induced in the flux allow one to distinguish the Majorana or Dirac nature of neutrinos, as well as the type of neutrino mass hierarchy (normal or inverted or quasi-degenerate). In some region of parameter space the determination of the absolute values of the neutrino masses is also conceivable. Measurements of the presence of these effects may be possible at the next-generation water Cerenkov detectors enriched with Gadolinium, or a 100 kton liquid argon detector.
\end{abstract}

\section{INTRODUCTION}

Neutrino flavor conversion has been observed in the solar (SuperK, SNO) [1], atmospheric (SuperK) [2], and terrestrial (KamLand, K2K) [3] neutrino data, providing evidence for nonvanishing, sub-eV neutrino masses. There now remains the longstanding theoretical question of how the neutrinos acquire their masses. The most elegant solution to this puzzle is the seesaw mechanism [4]: one assumes that lepton number is violated at some high scale $\Lambda_{\mathrm{L}}$ in the form of right-handed neutrino, $N$, Majorana masses, $M_{N} \sim \Lambda_{\mathrm{L}}$. This induces, at a lower 
scale, an effective operator of the form $\mathcal{O}(1) \times(L H)^{2} / \Lambda_{\mathrm{L}}$, where $L$ denotes a lepton doublet and $H$ the Higgs field. The oscillation data then imply that $\Lambda_{\mathrm{L}} \sim 10^{14} \mathrm{GeV}$. However, it is difficult to devise an experimental test of this mechanism (see however [5]). Therefore, it is important to explore alternate natural mechanisms for neutrino mass generation, especially those that may be tested in experiments at low energies.

A class of such models that have astrophysical and cosmological tests are the models of late-time neutrino mass generation [6, 7, 8]. In these models, neutrino masses are protected by some flavor symmetry different from the one related to the charged fermion masses, for example some global $U(1)_{N}$ symmetry. The small neutrino masses are generated when the new symmetry is broken at low scales. The effective Lagrangian for these models can be schematically written for either Dirac or Majorana particles where the neutrino fields are neutrino mass eigenstates as

$$
\mathcal{L}_{\nu}^{D}=\mathcal{L}_{k i n}+y_{\nu} \phi \nu N+V(\phi), \quad \mathcal{L}_{\nu}^{M}=\mathcal{L}_{k i n}+y_{\nu} \phi \nu \nu+V(\phi),
$$

where $\mathcal{L}_{k i n}$ is the kinetic piece of the lagrangian, $y_{\nu}$ is a dimensionless coupling, $\nu$ is a standard model neutrino field, $N$ is an extra field introduced for the case of neutrinos being Dirac particles, $\phi$ is the scalar field and $V(\phi)$ is the associated scalar potential. After spontaneous symmetry breaking the neutrinos acquire masses given by

$$
m_{\nu}=y_{\nu} f
$$

where $m_{\nu}$ is the mass of a particular neutrino mass eigenstate and $f$ is the symmetry breaking scale $(f=\langle\phi\rangle$, where $\langle\phi\rangle$ is the vacuum expectation value (VEV) of $\phi)$. With just one scalar the couplings are diagonal in the mass basis. In addition, since the scalar couples to neutrinos only, the constraints on the symmetry breaking scale $f$ are weak [7].

In addition to generating neutrino mass through their VEVs, the new light scalars provide another neutrino-neutrino interaction process aside from the Standard Model $Z^{0}$ exchange. The effects of the neutrinos coupling to these scalars on the cosmic microwave background has been previously studied [7]. Constraints have been placed on the symmetry breaking scale, $f$, the scalar mass, $M_{G}$, and the scalar-neutrino couplings, $y_{\nu}$, in these models from cosmological considerations [7, 8, 9], as well as from demanding that supernova cooling and the flux of the 1987a neutrinos would not be significantly modified in the presence of the additional fields [10, 11] (for constraints related to generating the observed baryon asymmetry of the universe see [12]). 
In this paper we show that the presence of this new physics significantly modifies the spectrum of supernova relic neutrinos (SRNs) at earth. This modification occurs because SRNs can interact with cosmic background neutrinos through exchange of the new light scalar. In [11] this effect was studied assuming a single flavor, Majorana, case. Here we extend our study and include various interesting aspects related to the nature of the neutrino flavor sector, for example the breaking of lepton number, the effect of multiple generation, etc. For simplicity we confine our study here to late neutrino mass models with a single $U(1)$ symmetry. We show that the energy spectrum of the SRN flux is sensitive to the type of neutrino mass hierarchy and whether neutrinos are Majorana or Dirac particles. We discuss how in some specific cases one can get additional information about the neutrino masses as well. In addition, detection of this signal would also be direct evidence of the presence of the cosmic background neutrinos. Hundreds of events per year from the flux of the SRN antineutrinos could be seen at next-generation large megaton water Cerenkov detectors [13, 14] such as UNO, Hyper-Kamiokande or MEMPHYS if they are enriched with Gadolinium [15], or from the flux of the SRN neutrinos in a large 100 kton liquid argon neutrino detector [16].

In section [I we show how the SRN flux, including cosmological evolution, is modified through the new interactions. In section [II] we consider the normal and inverted neutrino mass hierarchy cases as well as quasi-degenerate neutrino masses. We also consider the possibility of neutrinos being either Majorana or Dirac particles. In addition we show that there is a particularly interesting signal that leads to the determination of ratios of neutrino masses. The conditions for establishing a statistically significant signal above background are discussed in subsection G of section III. Finally, in section [V], we conclude.

\section{THE SUPERNOVA RELIC NEUTRINO FLUX}

The resonance interaction of the SRN with cosmic background neutrinos through the exchange of a new light scalar was previously discussed in [11] for a single neutrino mass eigenstate with $m_{\nu} \sim 0.05 \mathrm{eV}$.

In this paper we consider the case of the scalar interacting with three neutrino mass eigenstates and show the very interesting effect on the observed SRN spectrum for the normal mass hierarchy, inverted mass hierarchy, and for quasi-degenerate neutrino masses. 
We briefly discuss the case of adding one sterile neutrino.

We start with the SRN flux without the new interactions. The diffuse SRN flux is a remnant of neutrinos emitted from all the supernova that have occurred in the universe [17]. This flux is given by

$$
F\left(E_{\nu}\right)=\int_{0}^{z_{\max }} R_{S N}(z) \frac{d N\left((1+z) E_{\nu}\right)}{d E_{\nu}}(1+z)\left|c \frac{d t}{d z}\right| d z
$$

where $R_{S N}(z)$ is the comoving rate of supernova formation, $d N\left((1+z) E_{\nu}\right) / d E_{\nu}$ is the neutrino energy spectrum emitted by supernova, $d t / d z$ is for the cosmological expansion, $c$ is the speed of light, $z$ is the redshift, and $E_{\nu}$ is the neutrino energy.

The quantity $R_{S N}$ is the comoving rate of supernova formation, which can be parameterized as [17]

$$
R_{S N}(z)=\left(\frac{0.013}{M_{\odot}}\right) \dot{\rho}_{*}(z),
$$

where $\dot{\rho}_{*}(z)$ is the star formation rate given by

$$
\dot{\rho_{*}}(z)=(1-2) \times 10^{-2} M_{\odot} y r^{-1} M p c^{-3} \times(1+z)^{\beta} .
$$

We take $\mathrm{R}_{\mathrm{SN}}(0)=2 \times 10^{-4} \mathrm{yr}^{-1} \mathrm{Mpc}^{-3}, \beta=2$ (for $0<z<1$ ) and $\beta=0$ (for $z>1$ ) [17]. These are 'median' values for the parameters which have uncertainties in them coming from the uncertainty in the present knowledge of the cosmic star formation rate [17, 18]. ${ }^{1}$ The factor $d t / d z$ is given by

$$
\frac{d t}{d z}=-\left[100 \frac{\mathrm{km}}{\mathrm{s} \mathrm{Mpc}} h(1+z) \sqrt{\Omega_{M}(1+z)^{3}+\Omega_{\Lambda}}\right]^{-1},
$$

with $\Omega_{M}=0.3, \Omega_{\Lambda}=0.7$ and $h=0.7$.

The energy spectrum of the neutrinos emitted by a supernova has been modeled by several groups [19, 20, 21]. One of the models leads to perfect equipartition of the energy radiated into each neutrino flavor [19]. A second model makes a detailed one dimensional calculation of all relevant neutrino processes in the collapsing star and uses a variety of supernova progenitor masses [21]. Another model proposed by Keil, Raffelt, and Janka [20] (KRJ model) performs their calculations using a MC simulation. These models give spectra which have a width narrower than that of a thermal spectrum (so-called "pinched" spectrum [22]). It also predicts an average energy for the muon and tau flavor neutrinos very close to

\footnotetext{
${ }^{1}$ Future SN observatories will have the power to significantly reduce the related uncertainties 14 ]
} 
the average energy of the electron antineutrinos. As an illustrative example we take the neutrino spectrum given by the KRJ model with the additional assumption that the total energy carried by each neutrino flavor is $L_{\nu_{e}}=L_{\bar{\nu}_{e}}=L_{\nu_{x}}=5 \times 10^{52}$ ergs, where $\mathrm{x}$ stands for the muon and tau neutrinos and antineutrinos. The KRJ energy spectrum of neutrino flavor eigenstates produced by a supernova is given by

$$
\frac{d N_{\nu_{\alpha}}\left(E_{\nu_{\alpha}}\right)}{d E_{\nu_{\alpha}}}=\frac{\left(1+\beta_{\nu_{\alpha}}\right)^{1+\beta_{\nu_{\alpha}}} L_{\nu_{\alpha}}}{\Gamma\left[1+\beta_{\nu_{\alpha}}\right]{\overline{E_{\nu_{\alpha}}}}^{2}}\left(\frac{E_{\nu_{\alpha}}}{\overline{E_{\nu_{\alpha}}}}\right)^{\beta_{\nu_{\alpha}}} \exp \left[-\left(1+\beta_{\nu_{\alpha}}\right) \frac{E_{\nu_{\alpha}}}{\overline{E_{\nu_{\alpha}}}}\right],
$$

with the $\overline{E_{\nu_{\alpha}}}$ representing the average neutrino energies and $\beta_{\nu_{\alpha}}$ characterizing the amount of spectral pinching. Here for simplicity we set the values of the parameters to [20] (for recent numerical studies see e.g. [23])

$$
\begin{array}{ll}
\nu_{e}: \beta_{\nu_{e}}=3.4 & \overline{E_{\nu_{e}}}=13.0 \mathrm{MeV}, \\
\bar{\nu}_{e}: \beta_{\bar{\nu}_{e}}=4.2 & \overline{E_{\overline{\nu_{e}}}}=15.4 \mathrm{MeV}, \\
\nu_{x}: \beta_{\nu_{x}}=2.5 & \overline{E_{\nu_{x}}}=15.7 \mathrm{MeV},
\end{array}
$$

and also neglect effects such as shock wave and turbulence [24].

Because of matter oscillation effects, neutrinos emerge from a supernova as coherent fluxes of mass eigenstates which we label as $F_{\nu_{i}}$, where $i=1,2$, or 3 represents the particular neutrino mass eigenstate [25].

If neutrino flavor evolution inside of the collapsing star is either fully adiabatic or fully non-adiabatic (the flavor evolution is adiabatic if the mixing angle $\sin ^{2} \theta_{13} \gtrsim 10^{-3}$ and nonadiabatic if $\sin ^{2} \theta_{13} \lesssim 10^{-5}$ ) then the energy spectrum of each neutrino mass eigenstate that leaves the surface of the star corresponds to the original energy spectrum of some particular neutrino flavor eigenstate at emission from the neutrinosphere, i.e., there is a one-to-one correspondence between each $d N_{\nu_{\alpha}}\left(E_{\nu_{\alpha}}\right) / d E_{\nu_{\alpha}}$ and some $d N_{\nu_{i}}\left(E_{\nu_{i}}\right) / d E_{\nu_{i}}{ }^{2}$. The original produced flux of some neutrino flavor at the neutrinosphere will be labeled as $F_{\nu_{\alpha}}^{0}$. Translated back into the flavor basis, the expressions for the $\nu_{e}$ and $\bar{\nu}_{e}$ fluxes emerging from a supernova can be written as

$$
\begin{aligned}
& F_{\nu_{e}}=P_{H}\left|U_{e 2}\right|^{2} F_{\nu_{e}}^{0}+\left(1-P_{H}\left|U_{e 2}\right|^{2}\right) F_{\nu_{x}}^{0}, \\
& F_{\bar{\nu}_{e}}=\left|U_{e 1}\right|^{2} F_{\bar{\nu}_{e}}^{0}+\left|U_{e 2}\right|^{2} F_{\nu_{x}}^{0},
\end{aligned}
$$

2 See, for example, Table 1, Fogli, et. al. 33. 
for the normal mass hierarchy and

$$
\begin{aligned}
& F_{\nu_{e}}=\left|U_{e 2}\right|^{2} F_{\nu_{e}}^{0}+\left|U_{e 1}\right|^{2} F_{\nu_{x}}^{0}, \\
& F_{\bar{\nu}_{e}}=\bar{P}_{H}\left|U_{e 1}\right|^{2} F_{\bar{\nu}_{e}}^{0}+\left(1-\bar{P}_{H}\left|U_{e 1}\right|^{2}\right) F_{\nu_{x}}^{0},
\end{aligned}
$$

for the inverted mass hierarchy, where $P_{H}\left(\right.$ and $\left.\bar{P}_{H}\right)=0$ for the adiabatic case and $P_{H}\left(\right.$ and $\left.\bar{P}_{H}\right)=1$ for the non-adiabatic case [25]. In the equations above, $\left|U_{e 1}\right|^{2}=$ $\cos ^{2} \theta_{12},\left|U_{e 2}\right|^{2}=\sin ^{2} \theta_{12}, \theta_{12}=\theta_{\odot}\left(\theta_{\odot}\right.$ is the solar mixing angle [26] $)$, where $\sin ^{2} 2 \theta_{12}=$ $0.86 \pm 0.3$, and $\left|U_{e 3}\right|^{2} \approx 0$ [27]. When supernova neutrino flavor evolution is non-adiabatic then the $\nu_{e}$ and $\bar{\nu}_{e}$ flux for the normal and inverted hierarchies are identical.

We show that the addition of a new light scalar opens the possibility of determining the neutrino mass hierarchy independent of the neutrino fluxes, and also independent of the adiabatic or non-adiabatic nature of supernova neutrino flavor evolution.

\section{A. Modifications due to New Physics}

We consider the modifications to the SRN flux due to the resonance interaction of an SRN with a neutrino in the cosmic neutrino background. In this process a supernova neutrino with energy $E_{\nu}^{S N}$ will go through the resonance when the kinematic condition

$$
E_{\nu}^{S N}=\frac{M_{G}^{2}}{2 m_{i}} \equiv E_{i}^{R e s}
$$

is satisfied. More specifically, a neutrino observed with energy $E_{\nu}^{O b s}$ will have gone through resonance if its energy lies in the region

$$
\frac{E_{i}^{R e s}}{1+z}<E_{\nu}^{O b s}<E_{i}^{R e s},
$$

where $z$ is the redshift. There will be large depletion of the SRN flux in the energy domain given in Eq. 11] as long as the neutrino-scalar coupling satisfies [11]

$$
y>4.6 \times 10^{-8} \frac{M_{G}}{1 \mathrm{keV}} .
$$

This condition comes from requiring that the mean free path for absorption is much smaller than the Hubble scale. It is important to note that in the narrow width approximation for the resonance, the condition Eq. 12 is a sufficient condition to guarantee the absorption of all three neutrino flavors. After the neutrinos that have energies in the region given by Eq. 11 
go through the resonance they are redistributed to lower energies when the produced scalar decays back to neutrino mass eigenstates [28]. In particular, neutrinos after interaction will be redistributed with a flat energy distribution from zero energy up to the original energy of the incident supernova neutrino.

To find the effect of these interactions on the flux of the SRN we note that the neutrinos leaving a supernova at redshift $z$ emerge as the mass eigenstates. However, these mass eigenstate fluxes are now modified through interaction with the cosmic background neutrinos as they propagate to the earth. We consider for simplicity an Abelian U(1) late neutrino mass model. This implies that the Yukawa interaction between the scalars (in particular the Goldstone) and the neutrinos are diagonal in the mass basis (this is not the case in a model with non-Abelian symmetries). Supernova neutrinos are in their mass eigenstates and each mass eigenstate interacts only with the same mass eigenstate background neutrino via Goldstone exchange. To illustrate how the interactions modify the neutrino mass eigenstate flux we consider as an example the flux of the $\nu_{1}$ mass eigenstate, $F_{\nu_{1}}$.

We start by defining the modified flux of the $\nu_{1}$ eigenstates as $\widetilde{F_{\nu_{1}}}$. For each redshift $z$ the $\nu_{1}$ eigenstates that satisfy the condition given by Eq. 11 will have resonance interaction with cosmic background neutrinos, producing the intermediate scalar. A neutrino mass eigenstate will go through the resonance when the coupling satisfies Eq. 12. The cross section (averaged over the width of the resonance) for this to occur is approximately given by $\sigma_{R e s} \simeq \pi / M_{G}^{2}$. This will lead to a mean free path much smaller than the typical distance a supernova neutrino will travel to arrive at the earth, for the values of $M_{G}$ that we consider.

We label the absorbed flux as $F_{\nu_{1}}^{\text {Res }}$. Naively, the modified flux would be given by

$$
\widetilde{F_{\nu_{1}}}=F_{\nu_{1}}-F_{\nu_{1}}^{R e s}
$$

However, this expression does not take into account that the scalar decays back into neutrino mass eigenstates. We need to add this contribution to Eq. 13. The scalar can decay to any of the neutrino mass eigenstates. The probability that the scalar decays to a particular neutrino mass eigenstate is proportional to the square of the Yukawa coupling of that particular neutrino mass eigenstate to the scalar. From Eq. 1 we note that the relative probabilities are proportional to the ratios of squares of the neutrino masses,

$$
P_{j} \approx \frac{m_{j}^{2}}{\sum_{i=1}^{3} m_{i}^{2}} .
$$


Then the probability that a scalar decays to the neutrino mass eigenstate $\nu_{1}$ is $P_{1}$. These decays result in redistribution of the neutrino energies from zero energy up to the energy of the incident SRN with a flat energy distribution. We define $P_{1} \times F_{1 \rightarrow 1^{\prime}}^{R e s}$ as the fraction of the flux of $\nu_{1}$ that initiate a resonance, producing a scalar which then decays back into a $\nu_{1}$ eigenstate with degraded energy (indicated by the notation $1^{\prime}$ ). Then, Eq. 13 is modified to

$$
\widetilde{F_{\nu_{1}}}=F_{\nu_{1}}-F_{\nu_{1}}^{R e s}+P_{1} \times F_{1 \rightarrow 1^{\prime}}^{R e s}
$$

We still need to take into account the contributions from the decays of scalars produced by other neutrino mass eigenstates. Therefore, there should be a sum over all of the initial states, and Eq. 15 becomes

$$
\widetilde{F_{\nu_{1}}}=F_{\nu_{1}}-F_{\nu_{1}}^{\text {Res }}+P_{1} \sum_{i=1,2,3, \overline{1}, \overline{2}, \overline{3}} F_{i \rightarrow 1^{\prime}}^{\text {Res }}
$$

In more general notation, for the $j^{\text {th }}$ neutrino mass eigenstate,

$$
\widetilde{F_{j}}=F_{j}-F_{j}^{r e s}+P_{j} \times \sum_{i=1,2,3, \overline{1}, \overline{2}, \overline{3}} F_{i \rightarrow j^{\prime}}^{R e s} .
$$

The contributions over a range of redshift must be taken to determine the total flux at earth. If neutrinos are Dirac particles then there is factor of $1 / 2$ multiplying the last term (see discussion in Section IIIE).

The modified flux of electron neutrinos and electron antineutrinos can then be written as

$$
\widetilde{F_{\nu_{e}}}=\cos ^{2} \theta_{12} \widetilde{F_{\nu_{1}}}+\sin ^{2} \theta_{12} \widetilde{F_{\nu_{2}}}
$$

and

$$
\widetilde{F_{\bar{\nu}_{e}}}=\cos ^{2} \theta_{12} \widetilde{F_{\bar{\nu}_{1}}}+\sin ^{2} \theta_{12} \widetilde{F_{\bar{\nu}_{2}}}
$$

Finally, we note that each neutrino mass eigenstate goes through resonance at different energies given by Eq. 10 when there is just a single scalar of mass $M_{G}$. Depending on the details of the neutrino mass hierarchy, these resonance energies can either be very close to one another, or widely spaced apart.

\section{SIGNALS OF MODELS OF LATE-TIME NEUTRINO MASS GENERATION}

In this section we discuss the signals for the neutrino mass hierarchy in the observed SRN flux. We consider the case of the normal neutrino mass hierarchy, the inverted neutrino mass 
hierarchy, and the possibility that the neutrino masses are quasi-degenerate. We also show the effects of the neutrinos being Dirac or Majorana particles on the SRN flux signal. For these cases, unless otherwise noted, we choose the value of $E_{i}^{R e s}$, defined in Eq. 10, at $z=0$ to be equal to $15 \mathrm{MeV}$ for one of the neutrino mass eigenstates. This choice is made to illustrate the effects of the resonance process and to determine a region of the parameter space of the late-time neutrino mass generation models where the effect of the SRN modification would be seen. This is so, since for water Cerenkov detectors near reactors the background becomes negligible above about $13 \mathrm{MeV}$ [13, 14]. As will be discussed in the conclusions, the energy resolution is about $2 \mathrm{MeV}$. In subsections $\mathrm{A}$ through $\mathrm{E}$ we consider the case where neutrino flavor evolution in the $\mathrm{SN}$ is adiabatic, and in subsection $\mathrm{F}$ we show that the same features are obtained for a case where the flavor evolution is nonadiabatic. In subsection $G$ we discuss the detection of the new interactions.

In the following we focus on the flux of electron antineutrinos that arrive at earth, since the proposed water Cerenkov experiments for detection are sensitive to this neutrino flavor through the interaction of electron antineutrinos with protons with a cross-section given by [29]

$$
\sigma=10^{-43} p_{e^{+}} E_{e^{+}} E_{\nu}^{-0.07056+0.02018 \ln E_{\nu}-0.001953 \ln ^{2} E_{\nu}} \mathrm{cm}^{2},
$$

where $E_{e^{+}}$and $p_{e^{+}}$are the energy and momentum of the detected positron. Note that detection of the electron neutrino component of the SRN flux at a large liquid argon detector would provide complementary information [30].

\section{A. Normal Neutrino Mass Hierarchy}

As an example to illustrate how resonance interactions between the SRN and the cosmic background neutrinos can affect the SRN flux, we first consider a normal mass hierarchy of neutrino mass eigenstates. As a particular example of this hierarchy we choose the masses of the mass eigenstates to be

$$
\begin{aligned}
& m_{1}=0.002 \mathrm{eV}, \\
& m_{2}=0.009 \mathrm{eV}, \\
& m_{3}=0.05 \mathrm{eV} .
\end{aligned}
$$


This conforms to the best value of the atmospheric mass splitting $\left|m_{3}^{2}-m_{1,2}^{2}\right| \simeq 2.4 \times$ $10^{-3} \mathrm{eV}^{2}$ [31]. The value of the lightest mass, $m_{1}$, was chosen to be $0.002 \mathrm{eV}$ for the purpose of our numerical study, however there is no lower limit on the value of the mass of the lightest neutrino in either the normal or inverted hierarchies. If the mass of the lightest neutrino is lowered below the neutrino background temperature, $T_{C \nu B}$, then the resonance for this lightest state is governed by the corresponding thermal energy of the background neutrinos (see footnote 2 below). The particular choice for the neutrino masses in Eq. 21 results in the following features:

1. When a scalar is produced, it decays predominantly to the $m_{3}$ mass eigenstate. Eq. 14 gives $P_{3} \approx 0.967, P_{2} \approx 0.031$, and $P_{1} \approx 0.002$.

2. Because there is one scalar with mass $M_{G}$, Eq. 10 implies that the ratios of the neutrino masses govern the resonance energy positions, so that $E_{2}^{\text {Res }}=2 / 9 \times E_{1}^{\text {Res }}$ and $E_{3}^{\text {Res }}=1 / 25 \times E_{1}^{\text {Res }}{ }^{3}$

If we choose the lightest neutrino mass eigenstate to have the resonance at $15 \mathrm{MeV}$, to illustrate the effect, then the corresponding scalar mass is $M_{G} \approx 245 \mathrm{eV}$, and point 2 above implies that the other two resonances are $E_{2}^{\text {Res }} \approx 3.8 \mathrm{MeV}$ and $E_{3}^{\text {Res }} \approx 0.60 \mathrm{MeV}$. These two resonance energies are both well below experimental detection thresholds. Additionally, because the modified electron antineutrino flux is composed only of $\widetilde{\bar{\nu}_{1}}$ and $\widetilde{\bar{F}_{2}}$ the overall effect on $\widetilde{\bar{\nu}_{e}}$ is a depletion since decays of the scalar primarily contribute to $\widetilde{\bar{F}_{\bar{\nu}}}$. The resulting flux of electron antineutrinos can be seen in Fig. 1. Folding the flux with the cross-section for electron antineutrinos on protons,Eq. 20, gives the spectrum in Fig. 2 .

Relative to the electron antineutrino flux without interactions, the case with interactions has large depletion because of the dominant decay into the $m_{3}$ mass eigenstate. If we choose a heavier scalar so that the $m_{2}$ mass eigenstate goes through the resonance at 15 $\mathrm{MeV}$ instead of the $m_{1}$ mass eigenstate (i.e., $M_{G} \approx 490 \mathrm{eV}$ ), then we get the same feature, but the depletion is smaller. This is because $\widetilde{F_{\bar{\nu}_{1}}}$ is multiplied by $\cos ^{2} \theta_{12} \approx 0.70$, while $\widetilde{F_{\bar{\nu}}}$ is multiplied by $\sin ^{2} \theta_{12} \approx 0.30$, so $\widetilde{F_{\bar{\nu}}}$ is the smaller component of the final electron

3 The value of $E_{1}^{\text {Res }}$ for the lightest neutrino could be in the range $\sqrt{2 E_{\nu} T_{C \nu B}} \lesssim E_{1}^{\text {Res }} \leq \sqrt{2 E_{\nu} m_{1}}$, where the lower limit corresponds to the transition to the relativistic case and $T_{C \nu B}$ is the background neutrinos temperature. Since $T_{C \nu B} \sim 2 \times 10^{-4}$ which is not far from $m_{1}$ (given that the effect goes like the square root of the mass in that range) our results will only be slightly modified when this is taken into account. 


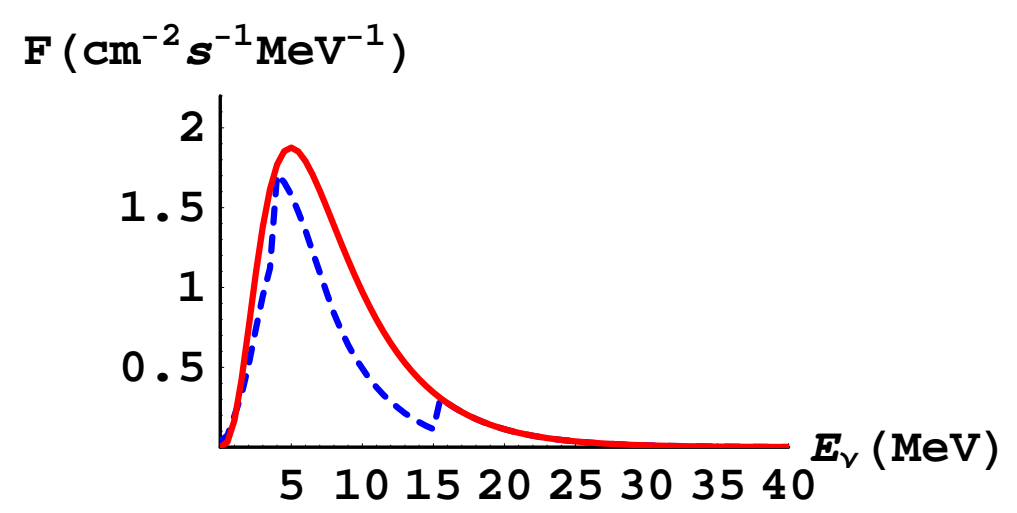

FIG. 1: The SRN electron antineutrino flux without interactions (red (solid) curve) and with interactions (blue (dashed) curve) when neutrinos are Majorana particles and for the normal mass hierarchy.

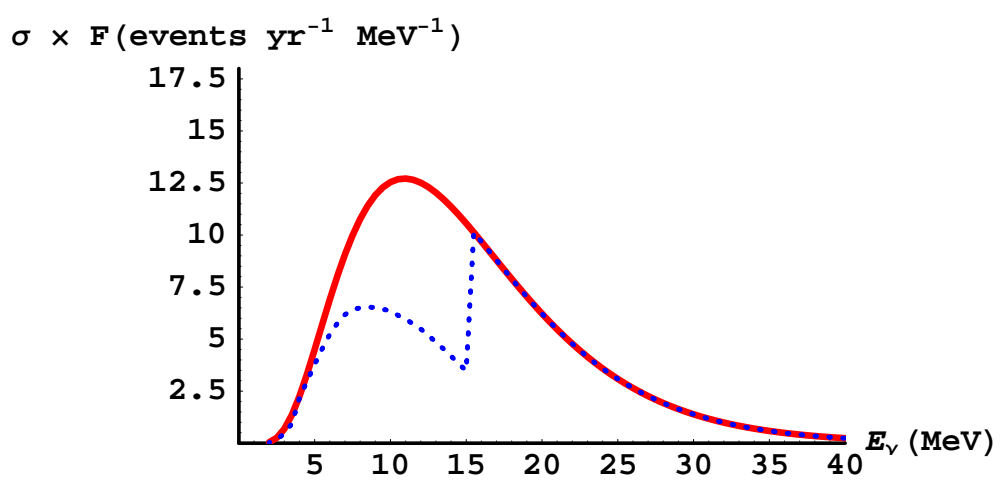

FIG. 2: The event rates for HyperKamiokande without interactions (red (solid) curve) and with interactions (blue (dashed) curve) when neutrinos are Majorana particles and for the normal mass hierarchy.

antineutrino flux. If the scalar were even heavier (i.e., $M_{G} \approx 1.2 \mathrm{keV}$ ) so that the $m_{3}$ mass eigenstate goes through the resonance at $15 \mathrm{MeV}$, then there is no depletion in the electron antineutrino flux since both the $m_{1}$ and $m_{2}$ eigenstates would have resonances at very high energies. As a result, neither of the corresponding fluxes would be visibly modified. 
The mass of the lightest neutrino can also be lowered without bound (although as mentioned above we do not consider the cases where the mass of the lightest neutrino is below the mass of the cosmic background neutrino temperature). For a fixed value of $M_{G}$, as the mass of the lightest state is lowered, the position of the resonance moves to higher energies until it is in a region where the flux of the SRN is too small for the feature to be experimentally observable.

To illustrate the effect on the electron neutrino spectrum, relevant to argon detectors such as Icarus and a future 100 kton argon detector [16], we show the event rates in Fig. 3 for the normal hierarchy case. A next generation liquid argon detector with a size of $100 \mathrm{ktons}$ could measure a significant number of electron neutrinos over just 5 years [16]. At neutrino energies lower than about $19 \mathrm{MeV}$ the solar neutrino flux dominates the SRN flux, and at energies greater than about $40 \mathrm{MeV}$ the atmospheric neutrino flux begins to dominate. In Fig. 3, to obtain event rates, we have folded the SRN flux with the cross-section for electron neutrinos to interact with argon [16]. We have used a resonance energy of $25 \mathrm{MeV}$ for the lightest neutrino mass state (taken to be $0.001 \mathrm{eV}$ so that $M_{G} \approx 225 \mathrm{eV}$ ). In this case we find significant reduction in the integrated event rate over the region in which the SRN flux is dominant.

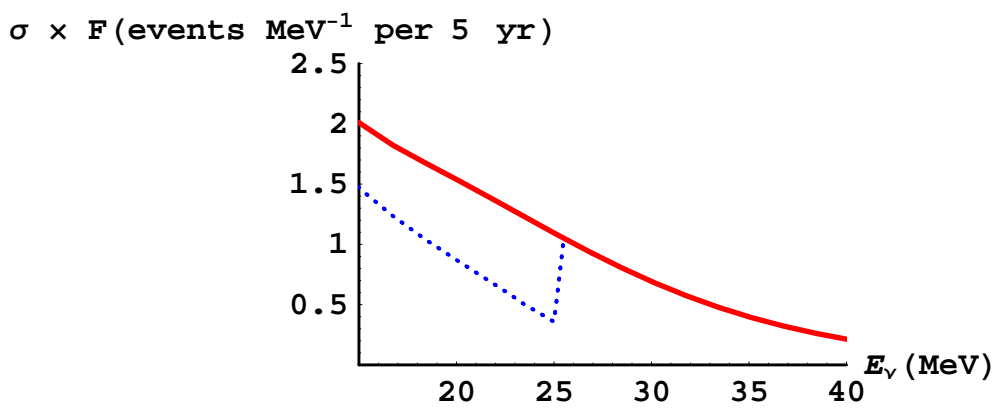

FIG. 3: The SRN electron neutrino flux for a normal hierarchy folded with the cross-section for electron neutrinos to interact with argon in a 100 kton detector running for 5 years. The red (solid) curve is for no interactions and the blue (dashed) curve is with interactions. 


\section{B. Inverted Neutrino Mass Hierarchy}

We now consider an example of the inverted mass hierarchy, characterized by $m_{1} \simeq$ $m_{2}>>m_{3}$, with neutrino masses chosen to be

$$
\begin{aligned}
m_{1} & =0.05 \mathrm{eV}, \\
m_{2} & \approx 0.05 \mathrm{eV}, \\
m_{3} & =0.008 \mathrm{eV} .
\end{aligned}
$$

This reflects the best value of $7.92 \times 10^{-5} \mathrm{eV}^{2}$ [31] for $\Delta m_{21}^{2}$, the $\nu_{1}-\nu_{2}$ mass splitting. Whenever a scalar is produced it dominantly decays into these two heavy eigenstates with

equal probabilities. Because $\widetilde{F_{\nu_{1}}}$ and $\widetilde{F_{\nu_{2}}}$ are the contributing components to the electron antineutrino flux, in this scenario there are both regions of depletion but also regions of overall enhancement of the flux due to rescattering. We choose the case where the $m_{1}$ and $m_{2}$ mass eigenstates go through resonance at $15 \mathrm{MeV}$, giving $M_{G} \approx 1.2 \mathrm{keV}$, and show the results in Fig. 4 (and Fig. $[5$ for weighting with cross-section).

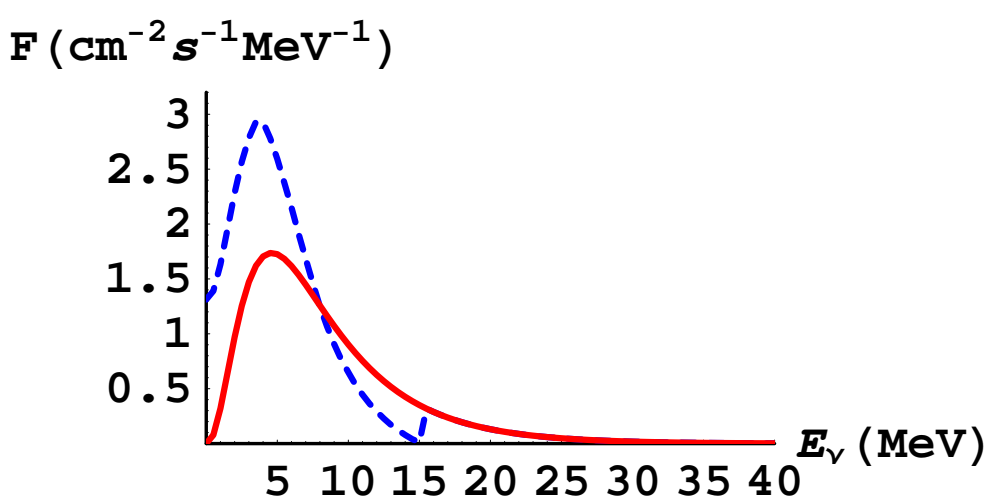

FIG. 4: The SRN electron antineutrino flux without interactions (red (solid) curve) and with interactions (blue (dashed) curve) when neutrinos are Majorana particles and for the inverted mass hierarchy.

We find that the enhancement is large because all initial neutrino mass eigenstate fluxes produce scalars which add to the low energy $m_{1}$ and $m_{2}$ eigenstate fluxes. The $m_{1}$ and $m_{2}$ eigenstate flux is depleted and is redistributed to lower energies. Once we fold the flux with 


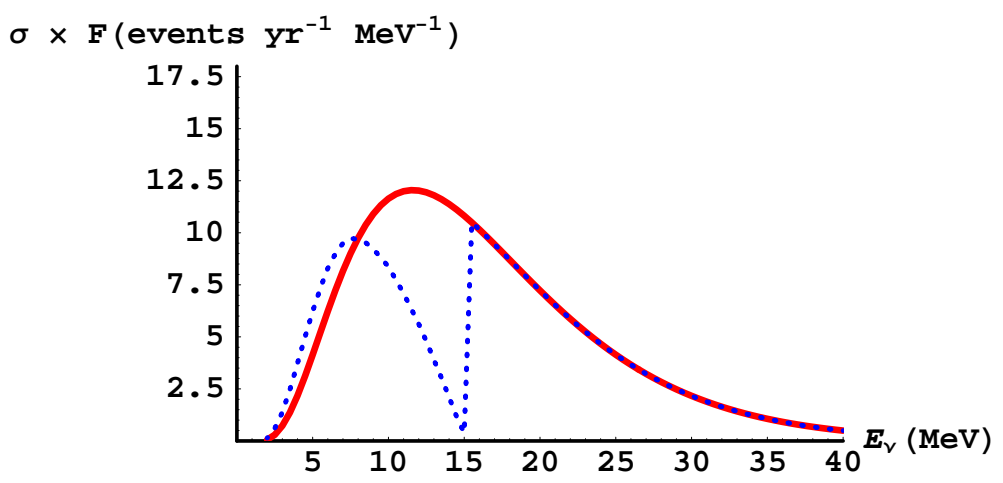

FIG. 5: The event rates for HyperKamiokande without interactions (red (solid) curve) and with interactions (blue (dashed) curve) when neutrinos are Majorana particles and for the inverted mass hierarchy.

the cross-section, we see in Fig. 5 that in contrast to the case of the normal mass hierarchy, the peak at low energies is more pronounced. This is a result of the electron antineutrino flux being composed only of the $m_{1}$ and $m_{2}$ neutrino mass eigenstates (since $\left|U_{e 3}\right|^{2} \approx 0$ ), both of which get depleted by the resonance at the same energy since the two eigenstates are nearly mass degenerate. Therefore the electron antineutrino flux is almost completely depleted near the resonance cutoff.

We apply similar analysis to the electron neutrino flux, of relevance to a liquid argon detector. In Fig. 6 we show event rates for 100kton liquid argon detector. We use a resonance energy of $25 \mathrm{MeV}$ for the two heavier mass eigenstates with mass $0.05 \mathrm{eV}$, which corresponds to $M_{G} \approx 1580 \mathrm{eV}$. The integrated event rate for energies above the solar background are reduced relative to the no interaction case. 


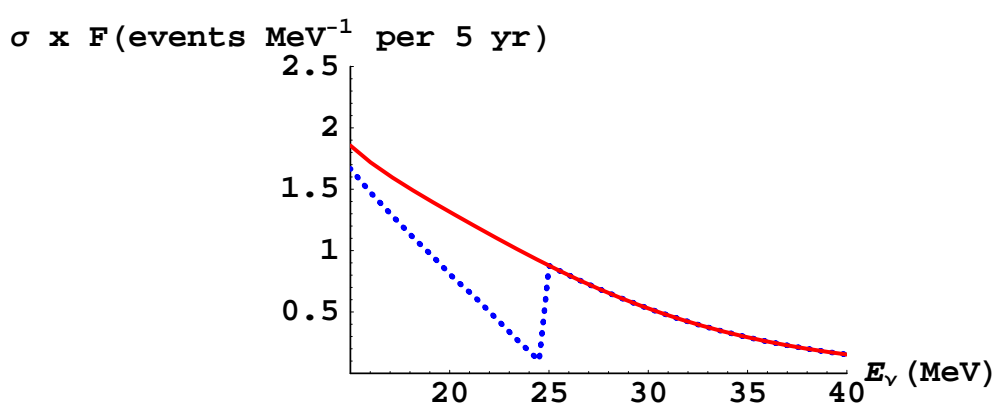

FIG. 6: The SRN electron neutrino flux for an inverted hierarchy folded with the cross-section for electron neutrinos to interact with argon in a 100 kton detector running for 5 years. The red (solid) curve is for no interactions and the blue (dashed) curve is with interactions.

\section{Quasi-Degenerate Neutrino Masses}

There still remains the possibility that the neutrino mass eigenstates are quasi-degenerate. For example, a mass hierarchy structure with

$$
\begin{aligned}
& m_{1} \approx 0.06 \mathrm{eV}, \\
& m_{2} \approx 0.06 \mathrm{eV}, \\
& m_{3} \approx 0.08 \mathrm{eV},
\end{aligned}
$$

satisfies the requirements for the two independent mass splittings as well as cosmological constraints on the sum of the neutrino masses [32]. We consider the case where the two eigenstates with mass $0.06 \mathrm{eV}$ have the same resonance energy as before, which would correspond to $M_{G}=1340 \mathrm{eV}$. The third mass eigenstate then has a resonance energy at approximately $11 \mathrm{MeV}$, however there is no corresponding dip since only the $m_{1}$ and $m_{2}$ mass eigenstates contribute to the final $\bar{\nu}_{e}$ flux. The result for the flux can be seen in Fig. 7 and for the event rate in Fig. 8 .

It is clear by comparing Fig. 4 and Fig. 7 that the case of the inverted mass hierarchy is nearly indistinguishable from the case of quasi-degenerate neutrino masses. However the quasi-degenerate case is distinguishable from the specific case of the normal mass hierarchy 


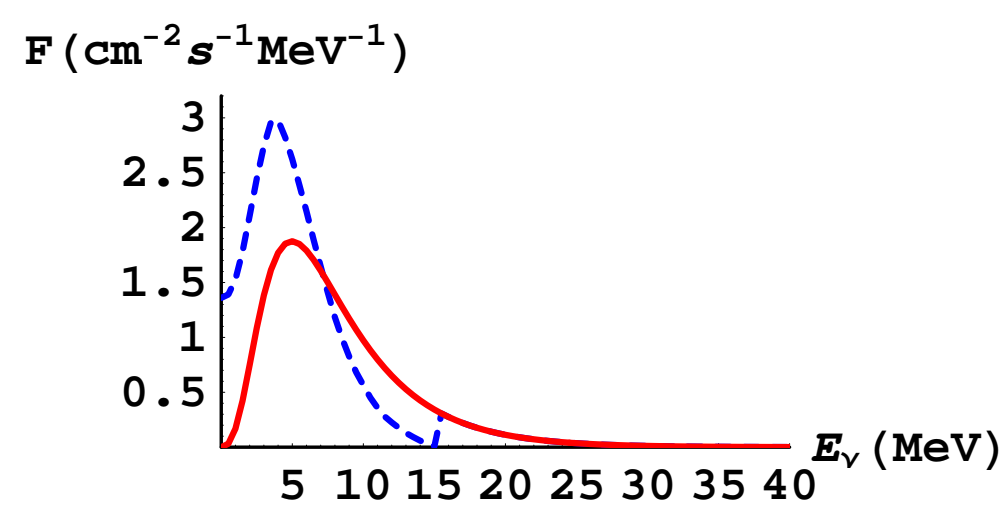

FIG. 7: The SRN electron antineutrino flux without interactions (red (solid) curve) and with interactions (blue (dashed) curve) when neutrinos are Majorana particles and for quasi-degenerate neutrino masses.

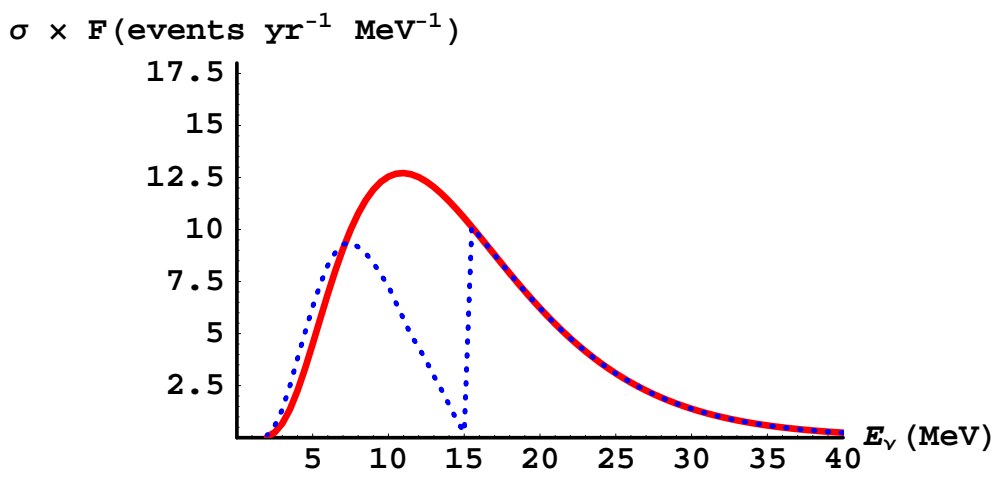

FIG. 8: The event rates for HyperKamiokande without interactions (red (solid) curve) and with interactions (blue (dashed) curve) when neutrinos are Majorana particles and for quasi-degenerate neutrino masses.

when one neutrino mass is much lighter than the other two masses. 


\section{Multiple Depletion Dips}

There is the possibility within the normal hierarchy that the $m_{1}$ and $m_{2}$ mass eigenstates are nearly, but not exactly, degenerate, and also still much lighter than the $m_{3}$ mass eigenstate. One example of the possible values for the masses in such a scenario is

$$
\begin{aligned}
& m_{1} \approx 0.01 \mathrm{eV}, \\
& m_{2} \approx 0.013 \mathrm{eV}, \\
& m_{3} \approx 0.05 \mathrm{eV}
\end{aligned}
$$

If we choose the resonance of the $m_{2}$ eigenstate to be at $15 \mathrm{MeV}$, then the $m_{1}$ mass eigenstate goes through resonance at $E_{1}^{\text {Res }} \approx 20 \mathrm{MeV}$. This corresponds to $M_{G} \approx 630 \mathrm{eV}$.

As can be seen in Fig. 9 and Fig. 10, this leads to two depletion dips in the final electron antineutrino spectrum and three peaks. There is always a signal corresponding to each neutrino mass eigenstate interacting with the Goldstone. The presence of two distinct depletion dips in an experimentally interesting region, however, is sensitive to the ratio of the masses of two of the neutrino mass eigenstates, in this case $m_{1}$ and $m_{2}$. For example, in Section IIA there is only one depletion dip because the ratio of the masses, and therefore the ratio of the resonance energies, for the $m_{1}$ and $m_{2}$ states is 4.5 , so that with $m_{1}$ resonance at $15 \mathrm{MeV}$ the $m_{2}$ resonance is at $3.8 \mathrm{MeV}$, outside of the observable region.

Experimental observation of the energy position of these dips could determine the ratio of the $m_{1}$ and $m_{2}$ masses, which together with the measured value of $\Delta m_{21}^{2}$ and $\Delta m_{32}^{2}$ allows one to determine the neutrino masses. This is a remarkable possibility since it is extremely hard to experimentally determine the exact values of the neutrino masses, especially the mass of the lightest state.

\section{E. Dirac vs. Majorana Neutrinos}

If neutrinos are Majorana particles, then each scalar decay produces a $\nu_{L} \nu_{L}$ or $\nu_{R} \nu_{R}$ for each mass eigenstate. If the neutrinos are Dirac particles then the scalar can decay to $\nu \bar{N}$ or to $N \bar{\nu}$, where $\bar{N}$ and $N$ are the extra neutrino fields added for the case of neutrinos being Dirac particles in the late-time neutrino mass generation models. Then only half of the decays of the scalar produce an antineutrino that will be seen in the detector. Therefore for 


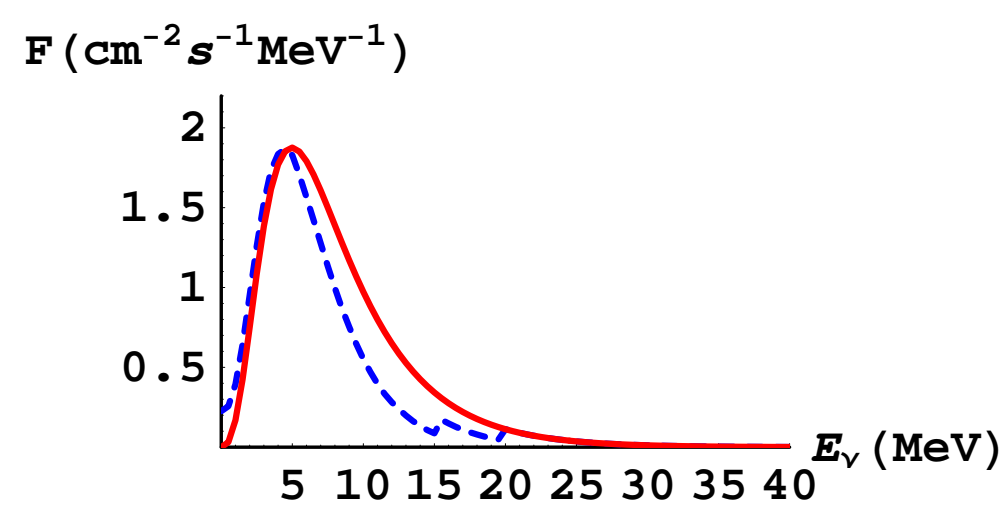

FIG. 9: The SRN electron antineutrino flux without interactions (red (solid) curve) and with interactions (blue (dashed) curve) when neutrinos are Majorana particles, for the normal mass hierarchy and where two neutrinos have distinct resonance features in the experimentally observable region.

the case of neutrinos being Dirac particles there is an overall factor of $1 / 2$ multiplying the last term of Eq. 17 relative to the case of neutrinos being Majorana particles.

If the neutrinos are arranged in a normal mass hierarchy as in Section II A then the ability to distinguish between the neutrinos being Dirac or Majorana particles is confounded by the small amount of scalar decays into the $m_{1}$ and $m_{2}$ eigenstates. However, in the case of the inverted mass hierarchy of Section IIIB there can be a visible difference in the electron antineutrino flux if the neutrinos are Dirac or Majorana particles as seen by comparing the Majorana particle case of Fig. 4 with the Dirac particle case of Fig. 11,

The resonance energy of the $m_{1}$ and $m_{2}$ mass eigenstates have been set to $15 \mathrm{MeV}$ in this case, exactly the same as for the Majorana particle case considered in Section IIIB. Because of the extra factor of $1 / 2$ the overall scale of the enhancement is much smaller than in the case of neutrinos being Majorana particles.

\section{F. Non-adiabatic case $\left(\sin ^{2} \theta_{13} \lesssim 10^{-5}\right)$}

If supernova neutrino flavor evolution is non-adiabatic, then the flux of electron antineutrinos that leaves a supernova is independent of the neutrino mass hierarchy (see 


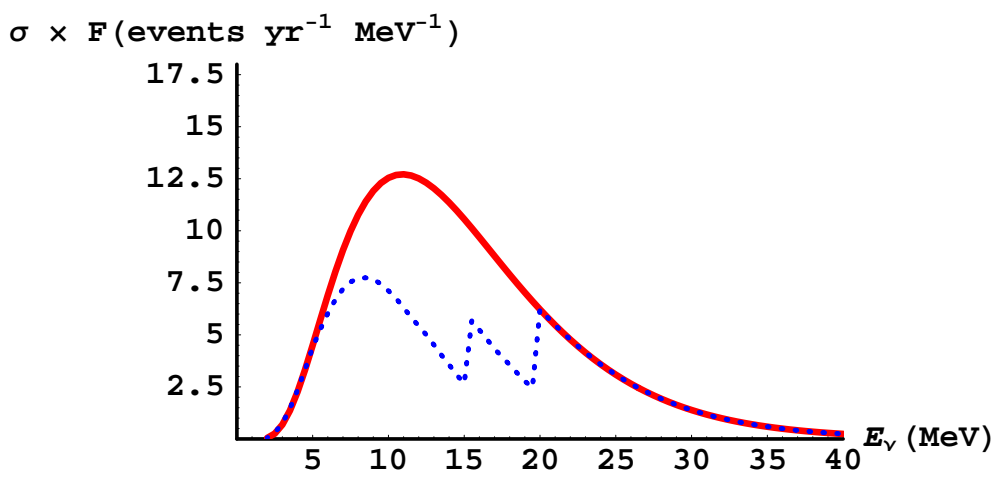

FIG. 10: The event rates for HyperKamiokande without interactions (red (solid) curve) and with interactions (blue (dashed) curve) when neutrinos are Majorana particles, for the normal mass hierarchy and where two neutrinos have distinct resonance features in the experimentally observable region.

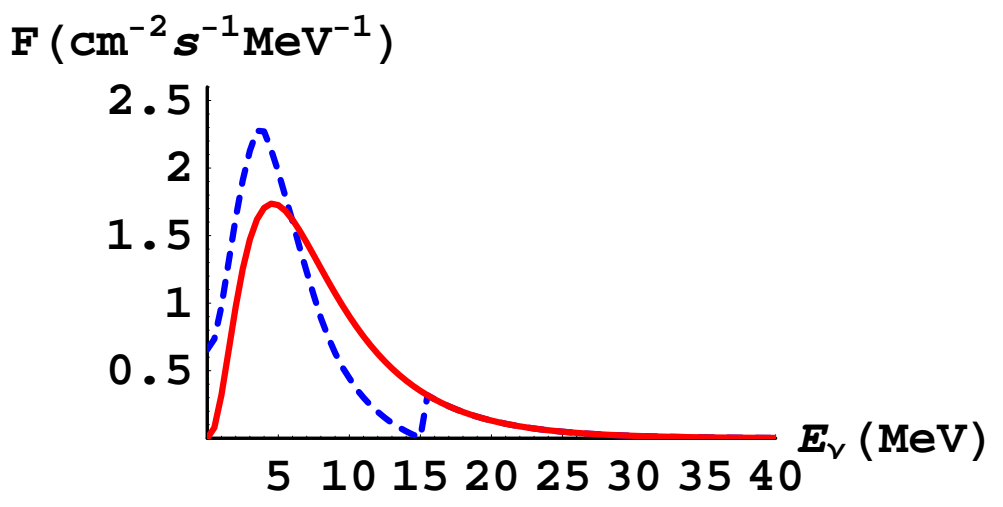

FIG. 11: The SRN electron antineutrino flux without interactions (red (solid) curve) and with interactions (blue (dashed) curve) when neutrinos are Dirac particles and for the inverted mass hierarchy.

Eq. 8 and Eq. 9). If the supernova neutrinos interact with the cosmic background neutrinos via new light scalars then the flux observed at earth will be different for the normal mass hierarchy and the inverted mass hierarchy. However, this difference is not detectable at 
the present or near-future neutrino experiments because it relies on the ability to observe neutrinos in the SRN flux at low energies where there is a large reactor background.

The difference between the two neutrino mass hierarchies is present, even in the case of small $\sin ^{2} \theta_{13}$, because for a normal mass hierarchy, the heavy neutrino mass eigenstate is $m_{3}$ which does not contribute to the $\nu_{\bar{e}}$ flux. All of the scalars produced through the neutrinoneutrino interactions will dominantly decay into this heaviest neutrino mass eigenstate, and the final flux will have an overall depletion relative to the SRN flux without interactions. However, for the inverted hierarchy, the $m_{1}$ and $m_{2}$ mass eigenstates are the heavy states, while the $m_{3}$ mass eigenstate is the light state. The scalars produced through the neutrinoneutrino interactions will dominantly decay into the two heavy states, leading to a low energy enhancement as well as the higher energy dip.

We show in Fig. 12 the SRN flux for the normal mass hierarchy (red/dotted curve) with $m_{1}=0.001 \mathrm{eV}, m_{2}=0.008 \mathrm{eV}, m_{3}=0.05 \mathrm{eV}$, and $M_{G}=173 \mathrm{eV}$, the inverted mass hierarchy (blue/dashed curve) with $m_{1}=0.05 \mathrm{eV}, m_{2}=0.05 \mathrm{eV}, m_{3}=$ $0.008 \mathrm{eV}$, and $M_{G}=1225 \mathrm{eV}$, and the SRN flux without interactions (black/solid curve).

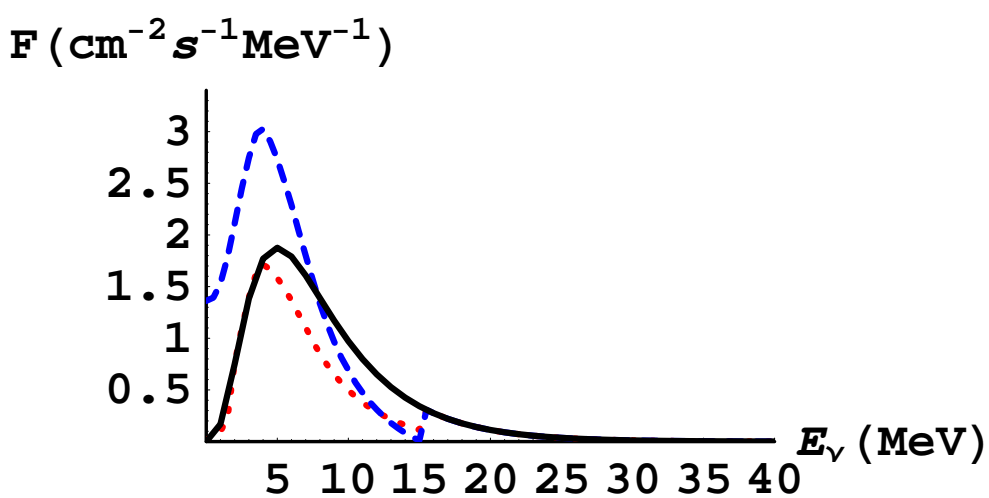

FIG. 12: The SRN electron antineutrino flux without interactions (black solid curve), with interactions and normal mass hierarchy (red dotted curve), and with interactions and inverted mass hierarchy (blue dashed curve), when neutrinos are Majorana particles and for $\sin ^{2} \theta_{13} \lesssim 10^{-5}$. 


\section{G. Signal Detection}

Here we show an example of an inverted mass hierarchy. The resonant energy for the $m_{1}$ and $m_{2}$ neutrino mass eigenstates is taken to be $16 \mathrm{MeV}$, which for $m_{1} \sim m_{2} \sim 0.05 \mathrm{eV}$ gives $M_{G} \sim 1265 \mathrm{eV}$. We present both the expected SRN flux as well as the SRN flux folded with the cross-section given in Eq. 20 in Fig. 13 and Fig. 14,

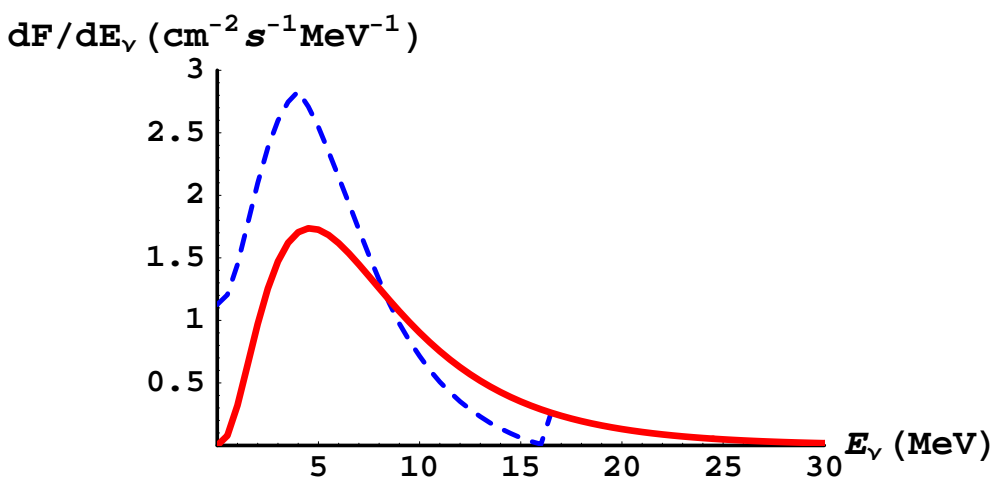

FIG. 13: The SRN electron antineutrino flux without interactions (red solid curve), and with interactions and inverted mass hierarchy (blue dashed curve) when neutrinos are Majorana particles.

Comparing the blue (dashed) curve to the red (solid) curve in Fig. 14 we see that there is a depletion of the SRN event rate above approximately $8 \mathrm{MeV}$ if the SRNs interact via the light scalar at resonance with the cosmic background neutrinos (blue dashed curve). This depletion is present up to the location of the dip, which in this case is $16 \mathrm{MeV}$. The main source of background, assuming the addition of Gd to the water Cerenkov detector, are nuclear reactor electron antineutrinos, but this background is small above neutrino energies of about $12 \mathrm{MeV}$ [13, 14]. This background is dependent on the location of the experiment and could be nearly absent. Clearly the detector which is not near nuclear reactors would have a better chance of seeing the signal for lower values of the resonance energy [13, 14].

To demonstrate the significance of our signal we look at an energy of $15 \mathrm{MeV}$ (where the effect is most significant and the reactor background is negligible). At this energy we expect approximately 11 events per year at HyperK from the SRN flux without new interactions 


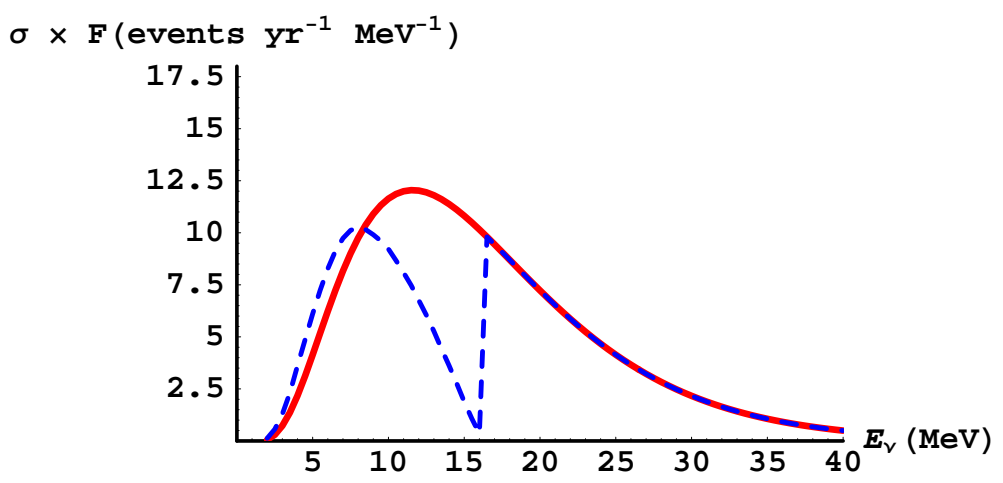

FIG. 14: The event rates for HyperKamiokande without interactions (red solid curve) and with interactions (blue dashed curve) when neutrinos are Majorana particles, for the inverted mass hierarchy.

(red solid curve). However, if the neutrinos interact via the light scalar at resonance, then we instead predict approximately 2 events per year (blue dashed curve) at this energy bin (assuming a $2 \mathrm{MeV}$ bin). The average fluctuation in the number of events expected in 5 years with no interactions can be estimated as $\sigma \sim \sqrt{55} \sim 7.5$ events. If we take as our signal the number of events expected without interactions minus the number of events expected with interactions (i.e., the event deficit) over the 5 year period (this is $55-10=45$ events), then in 5 years one expects approximately a $6 \sigma(45 / 7.5)$ effect. A similar analysis can be performed for the previous cases discussed in this paper, but since the depletion in these cases is at lower energies one must pay careful attention to the reactor background.

This analysis also requires a side band study, in order to determine the SRN flux in a region where interactions are ineffective (in our present example, this would be above $16 \mathrm{MeV}$ ). This would provide the overall normalization necessary for establishing the background. Clearly, once the shape of the signal without resonance and the non-SRN background are known, a more sophisticated analysis (including a bin-by-bin fit to the shape of the curves) can be achieved, and may even provide enhanced significance. This, however, is beyond the scope of this work, whose aim we regard to be a discussion of the qualitative aspect of our new physics signal.

For a liquid argon detector, if the resonance energy is above the cutoff for the solar 
neutrino flux at about $19 \mathrm{MeV}$, the integrated number of SRN electron neutrino events would be visibly reduced in the presence of new interactions. This is shown for a normal mass hierarchy and an inverted mass hierarchy in Fig. 3 and Fig. 6 respectively. While our total number of events is a conservative estimate (we use a $z$ evolution that is flat above $z=1$, instead of stronger dependence [16]), the depletion of events up to the resonance cutoff is a robust feature. We find that there is approximately a $25 \%$ reduction in the number of electron neutrino absorption events with new interactions compared to without new interactions for both the normal and inverted mass hierarchies for the energy window from $19 \mathrm{MeV}$ to $40 \mathrm{MeV}$.

\section{CONCLUSION}

The late-time neutrino mass generation models could be tested by detecting unique features of the SRN flux in both its electron antineutrino and neutrino components (for other tests of new physics that can be done with the SRN flux see [33]). To illustrate this new effect we have considered an Abelian U(1) model that generates neutrino masses at low scales. However, it is clear that the main features would still hold for a more complicated, nonAbelian model, although these models are already more constrained by BBN considerations. For example, one could still have observable dips in the SRN spectrum if the resonances are in a desirable energy window, but the couplings are no longer proportional to the neutrino masses, and so some predictive power is lost. However, one could hope to correlate the observations of the dip locations in the SRN spectrum with signals proposed to be present in the cosmic microwave background [7] for this case. We expect that the future generation water Cerenkov detectors enriched with gadolinium such as UNO, HyperKamiokande, or MEMPHYS would be able to detect a substantial number of SRN antineutrino events in a year [15]. Note that the threshold for this is on the order of $10 \mathrm{MeV}$ and depends on the location of the detector, especially due to reactor backgrounds [13, 14]. The effects of smearing due to the energy resolution of the water cerenkov-type detector needs to also be taken into account in a detailed analysis. For a Gaussian energy resolution function with width $\Delta(\Delta / \mathrm{MeV} \sim 0.6 \sqrt{E / \mathrm{MeV}})$, the smearing is only at most a few MeV [13] in the energy domain we considered. This smearing is then always smaller than the width of the depletion features considered. The neutrino component of the SRN flux could be detected 
by a large 100 kton liquid argon detector [16]. If there are neutrino-neutrino interactions through the light scalars present in these models, there is a possibility to distinguish between normal and inverted mass hierarchies and Dirac versus Majorana neutrinos, as well as to determine the absolute values of the neutrino masses. The ability to distinguish the neutrino mass hierarchy is independent of whether supernova neutrino flavor evolution is adiabatic or non-adiabatic.

The qualitative features of the signal of new interations via light scalar, such as the depletion, enhancement at lower energies, and the possibility to distinguish between neutrino mass hierarchy, as well as the nature of the neutrino are independent of the theoretical model for the supernova neutrino energy spectrum, which predict slightly different shape and wider range of average energies for different neutrino (antineutrino) flavors than the KRJ model [19, 20, 21]. This is not surprising because the produced neutrino spectrum does not depend on the detailed shape and normalization of the initial supernova neutrino fluxes but rather on the coupling of the scalar to the final neutrino mass eigenstates. As shown in section A (3) of the first paper [11], the presence of a deep dip is universal, and its position depends only on the masses of the scalar and the target neutrino, rather than any feature of the neutrino spectrum. For a normal neutrino mass hierarchy there is an overall depletion of the SRN flux, while for an inverted neutrino mass hierarchy there is an enhancement of the SRN flux at low energies and a region of depletion at higher energies. If a sterile neutrino with a much larger mass than the active neutrinos were also to couple to the new scalar, then independent of the details of the masses of the active neutrinos the effect would be almost complete depletion of the spectrum in some energy window since the scalar would decay predominantly to the massive sterile neutrino.

All of these signals, and especially their observation, depend on the parameters of the model. In Fig. 15 we show constraints on the parameter space for which the SRN effects can be obtained in the $y_{\nu}-M_{G}$ plane. The signals proposed here are present in the SRN flux only if the couplings of the neutrino mass eigenstates to the scalar are larger than the condition given in Eq. 12 for a given value of $M_{G}$. This condition comes from requiring that the mean free path for absorption of a SRN neutrino on a cosmic background neutrino is much smaller than the Hubble scale [11]. It is a sufficient condition to guarantee the absorption of all three neutrino flavors. This lower bound on the coupling is represented by the diagonal blue (solid) line. If the resonance energy is below $12 \mathrm{MeV}$ then there is a 
large background from nuclear reactor antineutrinos [13, 14]. To have a significant signal we take $M_{G}$ to be above $\sqrt{2 m_{\nu} E_{\nu, \text { min }}^{\text {Res }}}$, where $E_{\nu, \text { min }}^{\text {Res }}$ is approximately $15 \mathrm{MeV}$. These threshold values are represented by the three vertical red dashed lines which are calculated for values of $m_{\nu}=0.001 \mathrm{eV}, 0.008 \mathrm{eV}$, and $0.05 \mathrm{eV}$. If the mass of the scalar is larger than these values, then the signal would be above the reactor background. Similarly, the signal would not be observable if the mass of the scalar is large so that the heaviest neutrino mass eigenstate has a resonance energy in the region where the SRN flux is small. We also show the constraint imposed by BBN considerations, which is similar to the bound obtained from SN cooling and to the bound from the observation of undegraded SN1987A neutrino flux [11]. The SRN flux is also sensitive to the non-resonant process, for example $2 \nu \rightarrow \phi \rightarrow 2 G \rightarrow 4 \nu$, but only in a very small region of the parameter space, above the horizonal black dashed line and below the horizontal red solid line [11]. The area above the diagonal green dashed line corresponds to the BBN constraint for a non-abelian Majorana case. We note that there is still a large range of parameter space where the couplings are large enough to give SRN flux modification in an energy window that large neutrino detectors could directly probe.

We have shown that the cosmic background neutrinos interacting with supernova relic neutrinos through exchange of the light scalar lead to significant modification of the SRN flux observed at earth. These signals would be detectable for a large region of parameter space, in some cases at a significance of more than $5 \sigma$, and measurements of the presence of these effects are well within the reach of the next-generation water Cerenkov detectors enriched with gadolinium, or a large 100 kton liquid argon detector. Specifically we have shown that the changes induced in the flux by the exchange of the light scalars might allow one to distinguish between neutrinos being Majorana or Dirac particles, the type of neutrino mass hierarchy (normal or inverted or quasi-degenerate), and could also possibly determine the absolute values of the neutrino masses. An interesting feature is that the ability to distinguish neutrino mass hierarchy does not depend on the dynamics of the flavor evolution of neutrinos leaving the supernova (whether it is adiabatic or non-adiabatic), or on the specific shape and normalization of the initial supernova neutrino flux. Note that the hierarchy determination can be made by solely looking at the spectrum of supernova relic electron antineutrinos, without need to do the measurement of the flux of supernova relic electron neutrinos. In addition, the modification of the SRN flux in any of the proposed scenarios is a clear indication of the presence of the cosmic background neutrinos left over 
from the era of Big Bang Nucleosynthesis.

\section{Acknowledgements}

We thank Tom Weiler for many discussions and for his valuable comments and suggestions. This research was supported in part by DOE under contracts DE-FG0204ER41319 (JB and IS), DE-FG02-04ER41298 (JB and IS), DE-AC03-76SF00098 (GP)

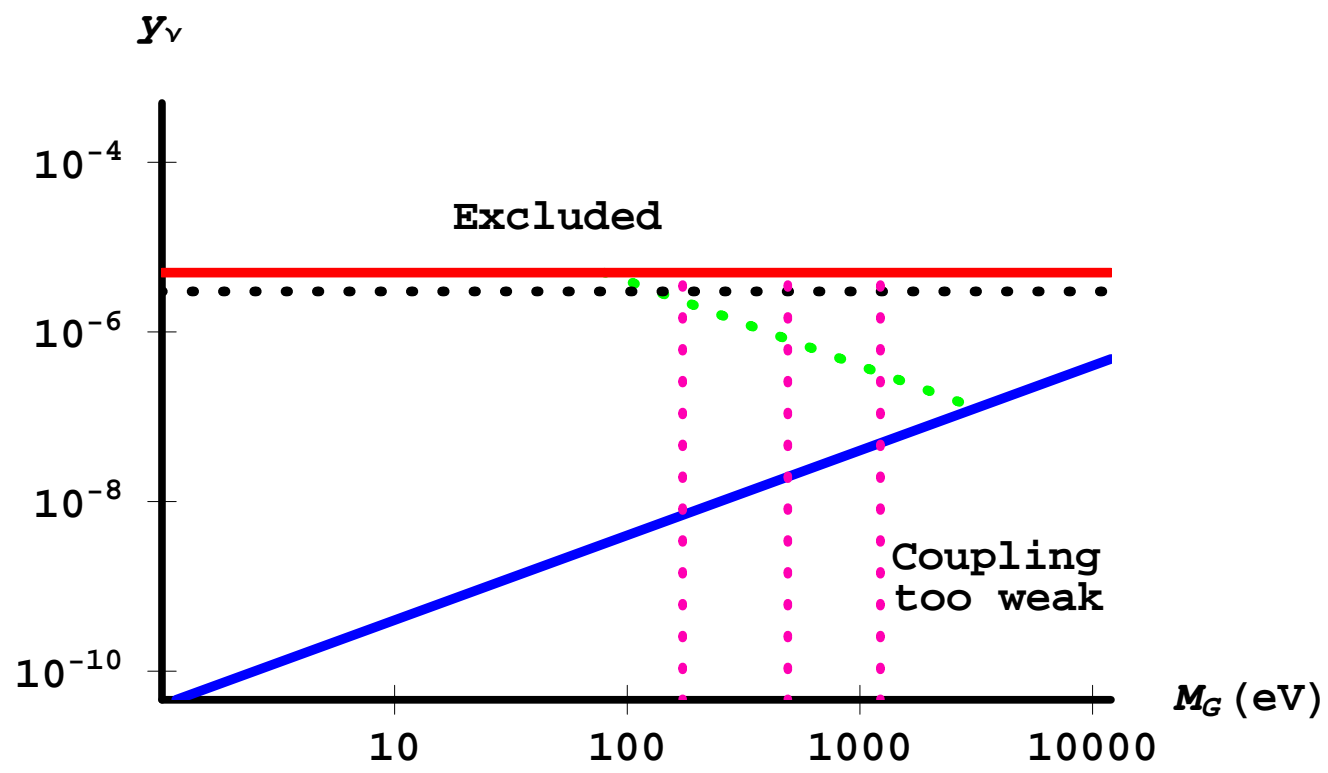

FIG. 15: The cosmological bounds and the regions for the supernova neutrino spectrum distortion due to the resonance and non-resonance processes for a single Majorana (Dirac) neutrino for an abelian (non abelian) model are shown in $\left(y_{\nu}, M_{G}\right)$ plane. The region above the red (solid horizontal) line is excluded by the BBN constraint (for the Dirac case), SN cooling (for Majorana case) and due to the observation of (undegraded) SN1987A neutrinos. In the region below the blue (solid slanting) line the mean free path is too long for the resonance to occur. The region above the green (dashed slanting) line, which is relevant only for the non abelian Majorana case, is the region excluded by the BBN constraint. The region above the black (dashed horizontal) line is the region of the future experimental sensitivity to the observation/non-observation of the SRN neutrinos due to non-resonant processes. The vertical dashed lines correspond to the minimum values of $M_{G}$ that lead to a depletion signal that is above the nuclear reactor antineutrino background for the neutrino masses of $0.001 \mathrm{eV}, 0.008 \mathrm{eV}$, and $0.05 \mathrm{eV}$ from left to right respectively. 
and NSF under grant PHY-0244507 (HG). JB and IS would like to thank LBL Theory Group for their hospitality while this work was being completed.

[1] B. T. Cleveland et al., Astrophys. J. 496, 505 (1998); Y. Fukuda et al., Phys. Rev. Lett. 81, 1158 (1998); J. N. Abdurashitov et al., J. Exp. Theor. Phys. 95, 181 (2002); Q. R. Ahmad et al., Phys. Rev. Lett. 89, 011301 (2002).

[2] Y. Fukuda et al., Phys. Rev. Lett. 81, 1562 (1998); Y. Ashie et al., ibid. 93, 101801 (2004).

[3] K. Eguchi et al., Phys. Rev. Lett. 90, 021802 (2003); M. H. Ahn et al., ibid. 90, 041801 (2003); T. Araki et al., ibid. 94, 081801 (2005); E. Aliu et al., ibid. 94, 081802 (2005).

[4] P. Ramond, M. Gell-Mann and R. Slansky, Supergravity, North-Holland (1979); T. Yanagida, in Proceedings of the Workshop on the Unified Theory and the Baryon Number in the Universe, Tsukuba (1979); R. N. Mohapatra and G. Senjanovic, Phys. Rev. Lett. 44, 912 (1980).

[5] M. R. Buckley and H. Murayama, Phys. Rev. Lett. 97, 231801 (2006).

[6] N. Arkani-Hamed and Y. Grossman, Phys. Lett. B459, 179 (1999).

[7] Z. Chacko, L. J. Hall, T. Okui and S. J. Oliver, Phys. Rev. D70, 085008 (2004); L. J. Hall and S. J. Oliver, Nucl. Phys. Proc. Suppl. 137, 269 (2004); Z. Chacko, L. J. Hall, S. J. Oliver and M. Perelstein, Phys. Rev. Lett. 94, 111801 (2005); T. Okui, JHEP 0509, 017 (2005).

[8] H. Davoudiasl, R. Kitano, G. D. Kribs and H. Murayama, Phys. Rev. D71, 113004 (2005).

[9] S. Hannestad, hep-ph/0602058; M. Cirelli and A. Strumia, JCAP 0612, 013 (2006).

[10] Y. Farzan, Phys. Rev. D67, 073015 (2003).

[11] H. Goldberg, G. Perez and I. Sarcevic, JHEP 0611, 023 (2006).

[12] L. J. Hall, H. Murayama and G. Perez, Phys. Rev. Lett. 95, 111301 (2005).

[13] J. F. Beacom and M. R. Vagins, Phys. Rev. Lett. 93, 171101 (2004); G. L. Fogli, E. Lisi, A. Mirizzi and D. Montanino, JCAP 0504, 002 (2005).

[14] L.J. Hall, H. Murayama, M. Papucci and G. Perez, hep-ph/0607109.

[15] C. K. Jung, AIP Conf. Proc. 533, 29 (2000); K. Nakamura, Int. J. Mod. Phys. A18, 4053 (2003); J. E. Campagne, M. Maltoni, M. Mezzetto and T. Schwetz, hep-ph/0603172.

[16] A. Ereditato and A. Rubbia, Nucl. Phys. Proc. Suppl. 154, 163 (2006).

[17] L. E. Strigari, M. Kaplinghat, G. Steigman and T. P. Walker, JCAP 0403, 007 (2004), S. Ando, et al., Astropart. Phys. 18, 307 (2003); M. Fukugita and M. Kawasaki, Mon. Not. 
Roy. Astron. Soc. 340, L7 (2003); S. Ando and K. Sato, Phys. Lett. B559, 113 (2003); S. Ando and K. Sato, New J. Phys. 6, 170 (2004); L. Strigari, et al., JCAP 0504, 017, (2005); H. Yuksel, S. Ando and J. F. Beacom, Phys. Rev. C 74, 015803, (2006).

[18] D. Schiminovich et al., Astrophys. J. 619, L 47 (2005); P. G. Perez-Gonzalez et al., ibid. 630, 82 (2005); A. M. Hopkins and J. F. Beacom, Astrophys. J. 651, 142 (2006); M. A. Fardal, N. Katz, D. H. Weinberg and R. Dav'e, astro-ph/0604534; F. Mannucci, H. Buttery, R. Maiolino, A. Marconi and L. Pozzetti, astro-ph/0607143.

[19] T. Totani, K. Sato, H. E. Dalhed and J. R. Wilson, Astrophys. J. 496, 216 (1998).

[20] M. Th. Keil, G. G. Raffelt and H. Th. Janka, Astrophys. J. 590, 971 (2003).

[21] T. A. Thompson, A. Burrows and P. A. Pinto, Astrophys. J. 592, 434 (2003).

[22] A. Mirizzi and G. G. Raffelt, Phys. Rev. D72, 063001 (2005); C. Lunardini, Astropart. Phys. 26, 190 (2006); C. Lunardini, Phys. Rev. D73, 083009 (2006).

[23] K. Kifonidis, T. Plewa, L. Scheck, H. Th. Janka and E. Mueller, astro-ph/0511369; C. L. Fryer, G. Rockefeller and M. S. Warren, Astrophys. J. 643, 292 (2006); A. Marek, H. Dimmelmeier, H. Th. Janka, E. Muller and R. Buras, Astron. Astrophys. 445, 273 (2006); A. Burrows, E. Livne, L. Dessart, C. Ott and J. Murphy, Astrophys. J. 640, 878 (2006); C. L. Fryer and A. Kusenko, Astrophys. J. Suppl. 163, 335 (2006); L. Scheck, K. Kifonidis, H. Th. Janka and E. Mueller, astro-ph/0601302.

[24] M. Rampp, R. Buras, H. Th. Janka and G. Raffelt, astro-ph/0203493; R. C. Schirato and G. M. Fuller, astro-ph/0205390 K. Takahashi, K. Sato, H. E. Dalhed and J. R. Wilson, Astropart. Phys. 20, 189 (2003); G. L. Fogli, E. Lisi, A. Mirizzi and D. Montanino, Phys. Rev. D68, 033005 (2003); R. Tomas et al., JCAP 0409, 015 (2004); G. L. Fogli, E. Lisi, A. Mirizzi and D. Montanino, ibid. 0606, 012 (2006); A. Friedland and A. Gruzinov, astro-ph/0607244.

[25] A. S. Dighe and A. Y. Smirnov, Phys. Rev. D62, 033007 (2000).

[26] M. C. Gonzalez-Garcia and Y. Nir, Rev. Mod. Phys 75, 345 (2003).

[27] S. Eidelman et al. [Particle Data Group], Phys. Lett. B592, 1 (2004).

[28] The decay is almost instantaneous compared to a Hubble time: the decay rate in the lab frame of the scalar produced at resonance is easily calculated to be $\sim\left(y^{2} / 16 \pi\right) m_{\nu}$, which is much larger than an inverse Hubble time if $\left.y \gg 4 \times 10^{-16}\left(1 \mathrm{eV} / m_{\nu}\right)\right)^{-1 / 2}$. In the light of the much stronger requirement Eq. 12, this is easily satisfied for all neutrino masses we consider.

[29] P. Vogel and J. F. Beacom, Phys. Rev. D60, 053003 (1999); A. Strumia and F. Vissani, Phys. 
Lett. B564, 42 (2003).

[30] A. G. Cocco, A. Ereditato, G. Fiorillo, G. Mangano and V. Pettorino, JCAP 0412, 002 (2004); J. F. Beacom and L. E. Strigari, Phys. Rev. C73, 035807 (2006); C. Lunardini, Phys. Rev. D73, 083009 (2006).

[31] G. L. Fogli, E. Lisi, A. Marrone, A. Palazzo and A. M. Rotunno, hep-ph/0506307.

[32] A. Goobar, S. Hannestad, E. Mortsell and H. Tu, JCAP 0606, 019 (2006).

[33] S. Ando, Phys. Lett. B570, 11 (2003); G. L. Fogli, E. Lisi, A. Mirizzi and D. Montanino, Phys. Rev. D70, 013001 (2004). These papers consider the effects of neutrino decay on the supernova relic neutrino flux observed at Earth. The neutrinos are coupled to very light or massless Majorons and the process considered is $\nu_{\text {Heavy }} \rightarrow \nu_{\text {Light }}+\phi$. 